\title{
FoxP2 Regulation during Undirected Singing in Adult Songbirds
}

\author{
Ikuko Teramitsu ${ }^{1}$ and Stephanie A. White ${ }^{1,2,3}$ \\ Interdepartmental Programs in ${ }^{1}$ Molecular, Cellular, and Integrative Physiology and ${ }^{2}$ Neuroscience and ${ }^{3}$ Department of Physiological Science, University of \\ California, Los Angeles, Los Angeles, California 90095
}

Learned vocal communication, including human speech, is a socially influenced behavior limited to certain animals. This ability requires auditory feedback during vocalization, which allows for on-line evaluation, to achieve the desired vocal output. To date, FOXP2 (forkhead box P2), a transcriptional repressor, is the only molecule directly linked to human speech. Identified FOXP2 mutations cause orofacial dyspraxia accompanied by abnormalities in corticostriatal circuitry controlling voluntary orofacial movements. These observations implicate FOXP2 in the developmental formation of neural circuits used in speech, but whether FOXP2 additionally plays an active role in mature circuitry was unknown. To address this question, we use a songbird, the zebra finch (Taeniopygia guttata), whose learned song and underlying circuitry are well characterized. We show that, when adult males sing, FoxP2 mRNA is acutely downregulated within area $\mathrm{X}$, the specific region of the songbird striatum dedicated to song. Furthermore, we find downregulation in males that sing by themselves (undirected singers) but not in males that sing to females (directed singers). This FoxP2 downregulation cannot be a simple consequence of the motor act because birds sang in both directed and undirected contexts. Our data suggest that FoxP2 is important not only for the formation but also for the function of vocal control circuitry. Social context-dependent, acute changes in FoxP2 within the basal ganglia of adult songbirds also suggest, by analogy, that the core deficits of affected humans extend beyond development and beyond basic central motor control.

Key words: basal ganglia; forkhead; language; speech; song; vocal learning; zebra finch

\section{Introduction}

To date, four distinct human cases forge the link between FOXP2 (forkhead box P2) and speech: the family known as KE, the individual CS, and two separate probands. In each case, affected individuals bear mutations in FOXP2 and suffer from speech problems (diagnosed as developmental verbal dyspraxia) (Lai et al., 2001; MacDermot et al., 2005; Zeesman et al., 2006). The KE family is the best characterized case. Of several linguistic features, their most prominent deficits are in sequencing of orofacial movements required for speech articulation, with no apparent deficiency in sequencing of manual movements (VarghaKhadem et al., 2005). FOXP2 expression in human embryonic brain coincides with regions of structural abnormalities in the affected individuals (Lai et al., 2003; Teramitsu et al., 2004) and maps to developing corticostriatal circuitry that later supports syntactic ability and speech production (Lieberman, 2002). These observations implicate FOXP2 in the development of neural

Received Feb. 7, 2006; revised June 5, 2006; accepted June 7, 2006.

This work was supported by Hyde Fellowship (I.T.), Alfred P. Sloan Foundation, University of California, Los Angeles Center for Autism Research and Treatment and Mental Retardation Research Center, National Alliance for Autism Research, Tennenbaum Creativity Initiative, and National Institutes of Health Grant MH070712 (S.A.W). We thank Justine Chen, Bill Luo, Amy Poopatanapong, and Tessa Watt for technical assistance and Drs. Arthur Arnold and Barney Schlinger for sharing resources. Dr. David Clayton provided a canary ZENK clone. Drs. Erin Schuman, Felix Schweizer, Alcino Silva, Spencer Smith, and three anonymous reviewers provided helpful comments.

Correspondence should be addressed to Stephanie A. White, 621 Charles E. Young Drive South, Los Angeles, CA 90095-1606. E-mail: sawhite@ucla.edu.

DOI:10.1523/JNEUROSCI.1662-06.2006

Copyright $\odot 2006$ Society for Neuroscience $\quad$ 0270-6474/06/267390-05\$15.00/0 structures used for speech. A similar view is proposed for Foxp2 mutant mice in which unlearned ultrasonic vocalizations are reduced (Shu et al., 2005).

Many Fox molecules specify cell lineage during embryogenesis yet have distinct functions in adults (Carlsson and Mahlapuu, 2002). FoxP2 is expressed in the mature brains of multiple species, including songbirds (Ferland et al., 2003; Teramitsu et al., 2004; Scharff and Haesler, 2005). In canaries, FoxP2 expression varies seasonally in area X (Scharff and Haesler, 2005), the subregion of songbird striatum specialized for song (Sohrabji et al., 1990). The persistent expression of FoxP2 into adulthood allows for a function in fully developed tissue. We therefore used zebra finches to test whether FoxP2 is actively regulated in adult corticostriatal circuitry during production of learned vocalizations.

Unlike canaries, zebra finches do not modify their songs seasonally but instead use the same song throughout adulthood. Zebra finch song nuclei are more stable in size than those of seasonal singers (Nottebohm, 1981; Thompson and Brenowitz, 2005). The use of adult zebra finches thus provides a rigorous test of whether any changes in FoxP2 expression are attributable to acute episodes of singing rather than to seasonal changes. Male finches display two types of singing behavior (Zann, 1996): “directed" singing occurs when males sing toward a conspecific during social interactions, and "undirected" singing occurs when males sing alone or in the presence of, but not toward, conspecifics and may serve as vocal practice (Jarvis et al., 1998). These two acoustically similar yet socially distinct behaviors are accompa- 
nied by distinct brain activation patterns (Jarvis et al., 1998; Hessler and Doupe, 1999). Analysis of FoxP2 in males singing directed versus undirected song could reveal whether any changes in neural expression reflect the basic motor act of singing or the social context in which singing occurs. We use these features of zebra finch song to demonstrate on-line regulation of FoxP2 mRNA in area $\mathrm{X}$ of adults. We observe this regulation during undirected, but not directed, singing. Our results suggest that FoxP2 has a postdevelopmental function within corticostriatal circuitry. Furthermore, the influence of social context on FoxP2 suggests that this regulation is not simply attributable to the motor act of singing, because birds were singing in both directed and undirected contexts.

\section{Materials and Methods}

Animals and tissues. Adult zebra finches (age $>100 \mathrm{~d}$ ) from our colony (14/10 h light/dark cycle) were used. After behavioral monitoring, birds were killed via decapitation for collection of brains. After rapid dissection, brains were frozen with liquid nitrogen and stored at $-80^{\circ} \mathrm{C}$ until use. All animal use was approved by the University of California, Los Angeles Institutional Animal Care and Use Committee.

Singing behavior and analyses. To test for any effects of episodic singing on FoxP2, we compared FoxP2 mRNA levels in area $\mathrm{X}$ of nonsinging males with those of males who sang in the presence of a female. Initially, we used females simply to encourage singing. We were not concerned about whether males directed all of their songs to the females. However, later analysis (see below) revealed that the social context of singing is crucial for detecting FoxP2 regulation and that, despite the female, most males sang songs that were not directed toward her. We refer to this group as "mixed singers" to indicate that their songs were neither $100 \%$ directed nor $100 \%$ undirected. We then used more rigorous methods (see below) to generate two additional groups of males that sang either $100 \%$ directed ("directed singers") or $100 \%$ undirected songs ("undirected singers"). To summarize, we used four groups: nonsingers, mixed singers, directed singers, and undirected singers. Experiments were conducted between 8:00 A.M. and 12:00 P.M. starting at lights on. Nonsinging birds were killed $2 \mathrm{~h}$ after lights on. Singing birds were killed between $2 \mathrm{~h}$ and $2 \mathrm{~h} 15 \mathrm{~min}$ after song onset in the morning, with one exception noted below.

Five nonsinging control males were kept from singing by the experimenter sitting nearby. Those that sang despite the experimenter's presence were excluded from additional analysis. For the mixed singers, five males were singly housed with a female. All but one of these males were killed at $2 \mathrm{~h}$ after song onset. The exception was killed $2 \mathrm{~h} 48 \mathrm{~min}$ after song onset. For the directed singers, six singly housed males were presented with one or two novel females every $3-4$ min over $2 \mathrm{~h}$ while their singing behavior, including body posture and orientation, was monitored. This frequent replacement of females resulted in performance of $100 \%$ directed songs. For undirected singing, 10 males singly housed were allowed to sing for $2 \mathrm{~h}$. Directed singers exhibited short bursts of intense singing during presentation with a new female, interspersed with quiet, whereas undirected singers sang freely in an undisturbed chamber. Thus, undirected singers sang more songs than directed singers.

Audio files were generated according to Livingston et al. (2000), and the number of motifs sung by each bird was counted. Sound Analysis Pro 1.04 software (Tchernichovski et al., 2000) was used to determine the degree of acoustic variability between syllables. Ten motifs per bird were analyzed for the within-syllable variability via 45 pairwise comparisons of the acoustic features using the local similarity measure (Olveczky et al., 2005). Resultant scores per syllable were then averaged for each bird.

Isolation of zebra finch FoxP2 truncated splice variant cDNA. We first tested whether zebra finches possess the two major alternative splice variants of FoxP2, a full-length form and a truncated form that lacks the forkhead DNA-binding domain identified previously in mammals (Shu et al., 2001; Bruce and Margolis, 2002). Indeed, we isolated the openreading frame (ORF) of a FoxP2 truncated splice variant from cDNA prepared according to Teramitsu et al. (2004) (GenBank accession num- ber DQ285023). Briefly, partial cDNAs of the truncated variant containing the $3^{\prime}$ portion of ORF and the $3^{\prime}$ untranslated region (UTR) were amplified by PCR using a set of primers designed based on the chicken genomic sequence of the corresponding variant (http://www.ensembl.org/Gallus_gallus/index.html) (sense, 5'-GCATCACCACCAATAACTCATC-3'; antisense, 5'-GCCGTATTTTTCATCACACTCA-3'). PCR cycling conditions (Advantage cDNA PCR; Becton Dickinson, San Jose, CA) were as follows: $2 \mathrm{~min}$ at $94^{\circ} \mathrm{C}$ for one cycle; and $15 \mathrm{~s}$ at $94^{\circ} \mathrm{C}$, $30 \mathrm{~s}$ at $51^{\circ} \mathrm{C}$, and $1 \mathrm{~min}$ at $72^{\circ} \mathrm{C}$ for 39 cycles. The entire ORF of the truncated variant was then obtained using a sense primer containing the known sequence of the zebra finch full-length FoxP2 spanning the region between 1 and $21 \mathrm{bp}$ and an antisense primer designed based on the obtained partial sequence for the zebra finch FoxP2 truncated variant, encompassing both coding and $3^{\prime}$ UTR (sense, 5'-ATGATGCAGGAATCTGCGACA-3'; antisense, 5'-CGTAGACCTATTTACTGTTT$\left.3^{\prime}\right)$. PCR conditions were as follows: $2 \mathrm{~min}$ at $94^{\circ} \mathrm{C}$ for one cycle; $15 \mathrm{~s}$ at $94^{\circ} \mathrm{C}, 30 \mathrm{~s}$ at $50^{\circ} \mathrm{C}$, and $2 \mathrm{~min}$ at $72^{\circ} \mathrm{C}$ for 35 cycles; and $3 \mathrm{~min}$ at $72^{\circ} \mathrm{C}$ for one cycle. Amplified cDNAs were subcloned, and seven individual clones were sequenced according to Teramitsu et al. (2004).

In situ hybridization analyses. To measure FoxP2 mRNA levels in zebra finch brain, in situ hybridizations were performed according to Teramitsu et al. (2004). Two distinct probes for FoxP2 were used to detect any differential regulation of the full-length versus truncated splice variants: one to the middle region shared by both variants (mid-probe) and the other to the $3^{\prime}$ region in the full-length variant $\left(3^{\prime}\right.$-probe). The truncated form is predicted to interfere with full-length function, by virtue of the dimerization domain. Heterodimerization between the two forms could destabilize FoxP2 binding to and repression of DNA. However, both probes yielded similar results. We thus primarily report results on fulllength FoxP2 obtained from the $3^{\prime}$-probe and only indicate data from both probes to emphasize the reliability of FoxP2 regulation by social context.

For quantification of FoxP2 expression, averaged optical density (OD) values were obtained from digitized images of autoradiograms from coronal brain sections. The background was subtracted from each image, and then the averaged OD in area $\mathrm{X}$ or adjacent striatum [striatum mediale (StM)] was measured using Adobe Photoshop 7.0 (Adobe Systems San Jose, CA). Images of adjacent Nissl-stained sections precisely overlaid on the film images enabled unbiased selection of the targeted area. To minimize variability between experiments, OD values obtained from area $\mathrm{X}$ were normalized to those from StM; the latter did not exhibit changes in gene expression as a function of experimental condition (data not shown). Ratios $\sim 1.0$ indicate that expression levels in area $\mathrm{X}$ are comparable with those in StM.

Undirected singing upregulates ZENK (acronym for zif-268, egr-1, NGFI-A, Krox-24) mRNA in area X. In canaries, one undirected bout induces a $\sim 90 \%$ increase in area X ZENK (Jarvis and Nottebohm, 1997), whereas levels remain low with directed singing (Jarvis et al., 1998). We therefore checked $Z E N K$ levels in area $\mathrm{X}$ of four of the five mixed singers as a marker for any undirected singing, despite the female presence. pBluescript SK vector containing 1887 bp canary ZENK cDNA fragment (Mello et al., 1992) was labeled with $\left[{ }^{33} \mathrm{P}\right] \mathrm{UTP}$ through in vitro transcription as with FoxP2 riboprobes. Brain sections adjacent to those used for FoxP2 analyses were used to determine ZENK expression.

The Brown-Forsythe test of homogeneity of variance was used to compare the variability of FoxP2 expression among the mixed singers with that among nonsingers or directed singers. The effect of song behavior (nonsinging, mixed, directed, or undirected) on FoxP2 expression was analyzed using the Kruskal-Wallis nonparametric test with significance set at $p<0.05$. Simple linear regressions were used to test whether amount of singing predicts FoxP2 levels.

\section{Results}

\section{FoxP2 levels in area $\mathrm{X}$ vary between singing adult males}

To first test for any effect of singing on FoxP2 expression, adult males in the mixed singing group were encouraged to sing for $2 \mathrm{~h}$ by individually housing them with a female. For comparison, nonsinging males were killed $2 \mathrm{~h}$ after lights on. In situ hybridiza- 
$\mathbf{a}$

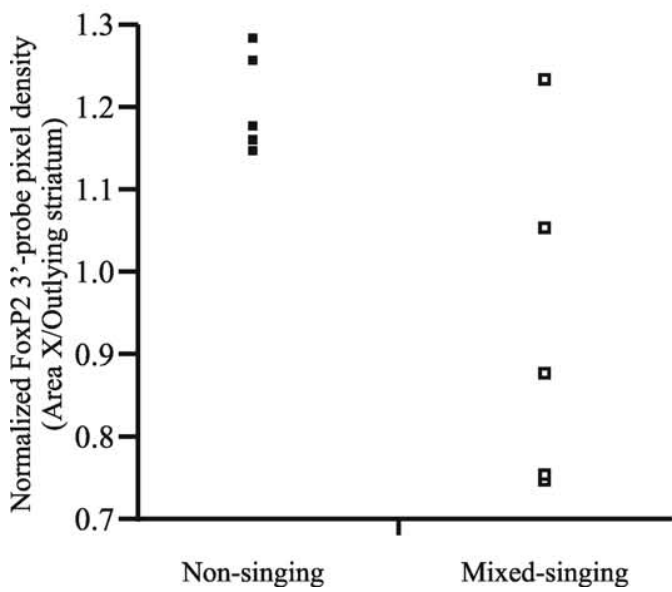

b

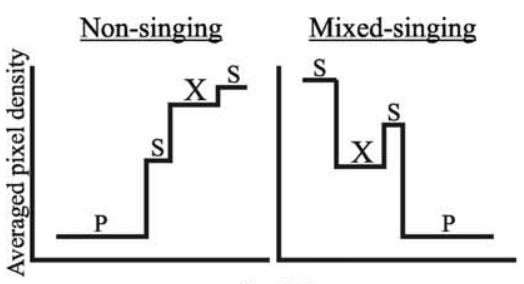

FoxP2

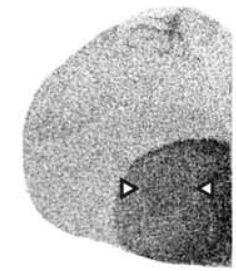

Non-singing

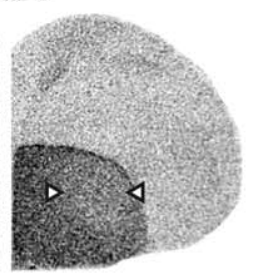

Mixed-singing c

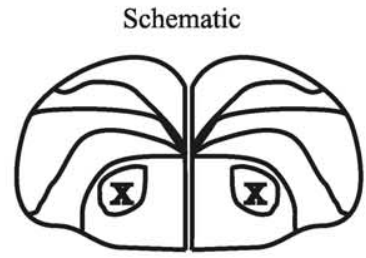

ZENK

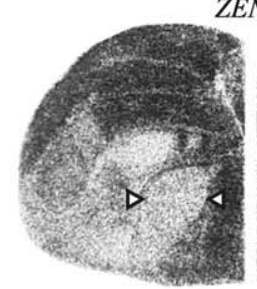

Directed-singing

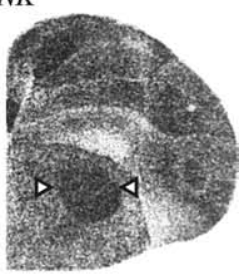

Mixed-singing

Figure 1. Area X FoxP2 levels vary between mixed singers. $a$, FoxP2 levels in area $X$ are similar to those of surrounding StM in nonsingers but appear downregulated in many mixed singers. The variability between mixed singers exceeds that between nonsingers $(p<0.05) \cdot \boldsymbol{b}$, Top, Average pixel density obtained from line scans through representative images of hemicoronal brain sections (bottom) depict FoxP2 levels measured at the widest mediolateral extent. FoxP2 levels in area $\mathrm{X}$ of the mixed singer that sang the fewest motifs (88 motifs; right) appear lower than those in the nonsinger (left). $\boldsymbol{c}$, High ZENK expression in area X of mixed singers (right; same bird as in $\boldsymbol{b}$, right) indicates that, although housed with females, males sang some undirected songs. Left image from a 100\% directed singer is shown for comparison. Schematic above indicates neuroanatomical landmarks. Arrowheads indicate area X. P, Pallium; S, striatum; X, area X.

tion analyses were performed on the collected brains using two distinct probes for FoxP2, 3'-probe and mid-probe. In the mixed singing group, we found high variability between birds in levels of FoxP2 in the striatal song nucleus, area X (SD of $0.21 ; n=5)$. This variability differed from the more uniform levels observed between nonsinging controls (SD of $0.06 ; n=5$; Brown-Forsythe test, $p=0.02$ ) (Fig. 1a). Of the five mixed singers, three showed visible downregulation of FoxP2 in area X compared with the surrounding StM.

Such downregulation was not observed in any of the nonsingers (Fig. 1b).

An interesting source of FoxP2 variability in the singing group could be a difference in the song types sung by each bird. Because mixed singers were individually housed with a female, they could have sung varying proportions of songs directed to her versus songs sung in an undirected manner. Undirected singing induces expression of the immediate early gene ZENK in area X (Jarvis et al., 1998), whose expression levels we therefore used to detect any undirected singing. We found robust ZENK expression in area $\mathrm{X}$ of all birds examined (four of five mixed singers), confirming that their songs were not entirely directed toward the females (Fig. 1c).

\section{FoxP2 is acutely downregulated in area $\mathrm{X}$ during undirected singing}

To discover whether the two socially distinct singing behaviors have differential effects on FoxP2 expression, we strictly controlled the social context of singing such that birds sang either $100 \%$ directed or $100 \%$ undirected songs. Indeed, our method of ensuring 100\% directed singing removed the variability of FoxP2 levels observed previously between the mixed singers (mixed, SD of $0.21, n=5$ vs directed, $\mathrm{SD}$ of $0.07, n=6$; Brown-Forsythe test, $p=0.013)$. We found a striking and reliable decrease in FoxP2 expression in area $\mathrm{X}$ of undirected singers $\left(3^{\prime}\right.$-probe, $0.87 \pm$ 0.037, mean \pm SEM; mid-probe, $0.87 \pm 0.028, n=10$ ) (Figs. 2,3 ) compared with directed and nonsingers. Such downregulation of

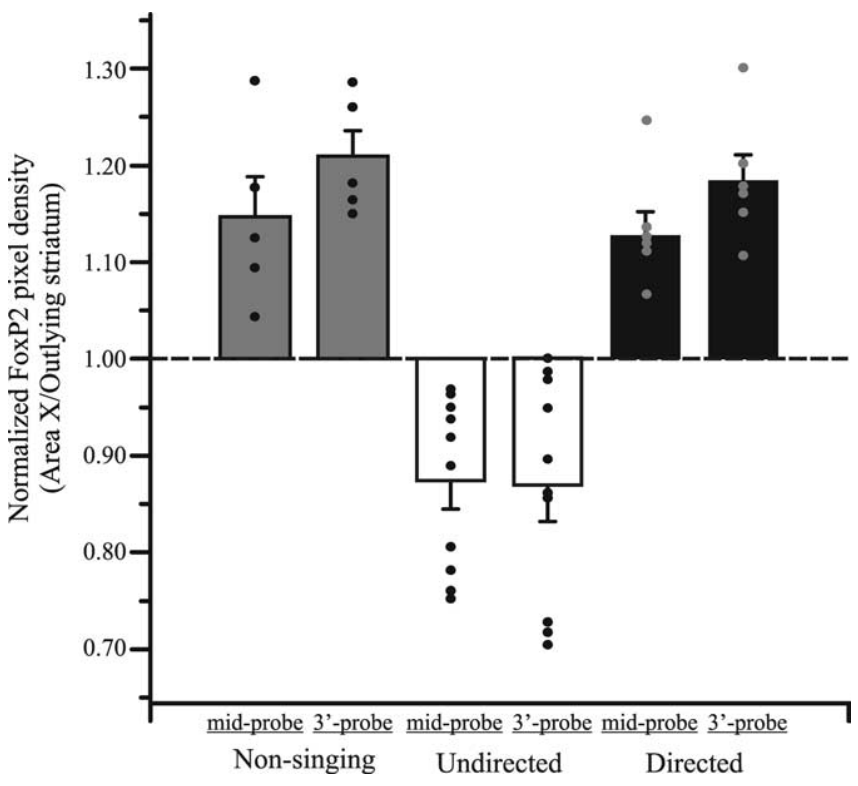

Figure 3. Undirected singing acutely downregulates FoxP 2 in area $X$. Bar graphs show group and individual values of FoxP2 expression in area X relative to surrounding StM (mean \pm SEM). Levels in undirected singers (white) are lower than in nonsingers (gray) or directed singers (black) (mid-probe, $p=0.0006 ; 3^{\prime}$-probe, $p=0.0005$ ). Nonsinging data are the same as shown in Figure 1. 


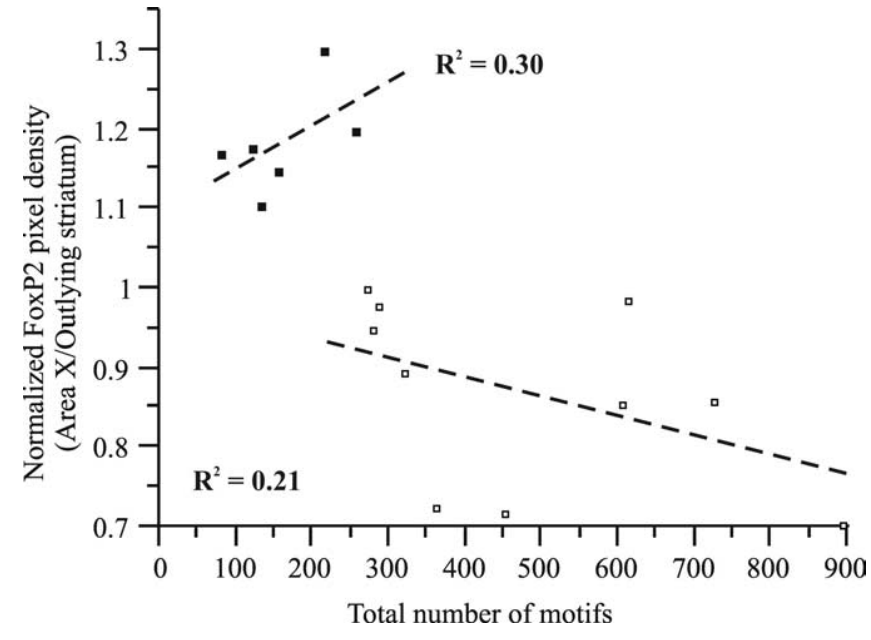

Figure 4. Trends in the correlation between FoxP2 and singing differ between directed (black; $p=0.26$ ) and undirected (white; $p=0.18$ ) singers.

FoxP2 was never observed in directed singers ( $3^{\prime}$-probe, $1.19 \pm$ 0.027 ; mid-probe, $1.13 \pm 0.024 ; n=6)$ or in nonsingers $\left(3^{\prime}\right.$ probe, $1.21 \pm 0.027$; mid-probe, $1.15 \pm 0.042)$. Similar results were obtained for both probes (Kruskal-Wallis test, 3 '-probe, $p$ $=0.0005$; mid-probe, $p=0.0006$ ) (Figs. 2, 3), indicating either parallel regulation for the two splice variants or that any independent changes in the truncated transcript are below our level of detection.

\section{Effects of social context}

Directed singers sang fewer motifs than undirected singers $(p=$ 0.0014; directed, range of 79-258, mean \pm SEM of $160.2 \pm 26.7$, vs undirected, range of $274-894$, mean \pm SEM of $481.7 \pm 68.6$ ), likely reflecting characteristics inherent to the behaviors (see Materials and Methods). Directed singers also had higher syllable similarity than undirected singers $(p=0.04$; directed, range of $87-91 \%$ similar, mean \pm SEM of $89 \pm 0.6 \%$ vs undirected, range of $82-90 \%$, mean \pm SEM of $86 \pm 0.9 \%$ ) as shown previously (Olveczky et al., 2005). Within the undirected singers, neither syllable similarity ( $3^{\prime}$-probe, $n=10 ; R^{2}=0.09 ; p=0.4$ ) nor the amount of singing correlated with the extent of FoxP2 downregulation (Fig. 4).

\section{Discussion}

FOXP2 has been directly linked to speech through four independent cases in which mutations in the FOXP2 coding sequence are accompanied by orofacial dyspraxia (Lai et al., 2001; Liegeois et al., 2003; MacDermot et al., 2005; Zeesman et al., 2006) and abnormalities in corticostriatal circuitry controlling voluntary orofacial movements (Liegeois et al., 2003). It seems clear that FOXP2 is required for development of circuits that later support speech (Liegeois et al., 2003). Whether FOXP2 is necessary solely for the formation of these circuits or also has an on-line role during their function was not known. Here, we used the zebra finch, a well characterized vocal learning model, to test for behavioral regulation of FoxP2 in adults during production of their learned songs. Within area $\mathrm{X}$, the striatal region specialized for birdsong (Sohrabji et al., 1990), we observe acute changes in FoxP2 levels after singing. Because both song learning and song circuit development is complete in adult zebra finches, our findings expand the focus of FoxP2 function from the formation to the actual use of such circuitry.

This on-line regulation of FoxP2 in adult zebra finches prompts the question of whether the human FOXP2 cases are purely developmental (Hurst et al., 1990; Watkins et al., 2002). Foxp2 is involved in lung, gut, heart, and brain development (Shu et al., 2001; Lai et al., 2003), yet the deficits of affected individuals appear confined to brain (Vargha-Khadem et al., 2005). Among Fox transcription factors, the FoxP subfamily has the unique ability to heterodimerize among its members to repress transcription of common gene targets (Li et al., 2004). Given that FoxP1 and FoxP4 are coexpressed with FoxP2 in lung (Lu et al., 2002) and brain (Ferland et al., 2003; Teramitsu et al., 2004; Scharff and Haesler, 2005), these molecules might compensate for one another developmentally. In contrast, FOXP1 and FOXP4 might be unable to substitute for a more immediate on-line function of FOXP2 in mature neural circuitry, producing a disorder restricted to brain function. The deficits in affected humans may thus reflect a lack of on-line function, in addition to developmental defects.

Our data also suggest that FoxP2 in adult zebra finches is regulated in a manner that depends not only on the motor act itself but also on the social context: during undirected singing, FoxP2 is downregulated in area $\mathrm{X}$, but, during directed singing, FoxP2 levels are stable. If FoxP2 were simply involved in vocalmotor production, we should have observed similar regulation by directed and undirected singing because the two behaviors involve the same motor apparati. We did not. The very site of regulation, area $\mathrm{X}$, is not thought to play a role in adult vocal production (Sohrabji et al., 1990). It is, however, a part of the anterior forebrain pathway, a specialized circuit within the avian equivalent of the mammalian cortico-basal-ganglia loop, which continues to modulate song on-line in adulthood (Kao et al., 2005). In undirected singing, neural activity increases and becomes more variable in area X (Hessler et al., 1999), accompanied by a subtle but significant increase in syllable variability compared with directed singing (Olveczky et al., 2005). Such variability is hypothesized to reflect the subtle motor exploration used in the reinforcement of learned song in adults (Doya and Sejnowski, 1995).

Downregulation of FoxP2, a transcriptional repressor, in area $\mathrm{X}$ during undirected singing might de-repress molecules related to exploratory behavior. We thus tested whether the amount of FoxP2 downregulation and the degree of motor stability were correlated in undirected singers. We found that undirected singers produce less stable, and thus more variable, syllables than directed singers, as shown previously (Olveczky et al., 2005). However, the syllable variability within our undirected singers did not correlate with the extent of FoxP2 downregulation. The small range of variability in these adult songs (82-90\%) may preclude detection of any such correlation. Future studies in young birds that produce more variable songs may be more revealing.

Undirected singers sang more than directed singers. However, this difference is unlikely to account for the difference in FoxP2 downregulation for four reasons: (1) directed singers sang on average $>88$ motifs, an amount that was sufficient for producing downregulation in a mixed singer (area X/StM OD ratio of 0.88 ) (Fig. 1b); (2) there was no correlation between the amount of singing and the amount of downregulation when birds sang under mixed $\left(n=5 ; R^{2}=0.28 ; p=0.36\right)$ or undirected conditions, despite the increased sample size for the undirected group $(n=$ $10 ; R^{2}=0.21 ; p=0.18$ ) (Scharff and Haesler, 2005); (3) a positive trend in the relationship between the number of motifs and FoxP2 levels observed in directed singers contradicts the negative trend in undirected singers (Fig. 4); and (4) there is precedence 
for social-context-dependent gene regulation in area X (Jarvis et al., 1998). Together, our data strongly suggest that FoxP2 is regulated in a social-context-dependent manner.

Our discovery of differential, on-line regulation of FoxP2 in the adult by two acoustically similar yet socially distinct vocal behaviors suggests roles for FoxP2 that extend beyond the development and simple use of vocal control circuitry. Although not obtainable from humans, these data, by analogy, suggest that the human cases of FOXP2 mutations involve postdevelopmental deficits in addition to developmental ones.

\section{References}

Bruce HA, Margolis RL (2002) FOXP2: novel exons, splice variants, and CAG repeat length stability. Hum Genet 111:136-144.

Carlsson P, Mahlapuu M (2002) Forkhead transcription factors: key players in development and metabolism. Dev Biol 250:1-23.

Doya K, Sejnowski TJ (1995) A novel reinforcement model of birdsong vocalization learning. In: Advances in neural information processing systems, Vol 7 (Tesauro G, Touretzky DS, Leen TK, eds), pp 101-108. Cambridge, MA: MIT

Ferland RJ, Cherry TJ, Preware PO, Morrisey EE, Walsh CA (2003) Characterization of Foxp2 and Foxp1 mRNA and protein in the developing and mature brain. J Comp Neurol 460:266-279.

Hessler NA, Doupe AJ (1999) Social context modulates singing-related neural activity in the songbird forebrain. Nat Neurosci 2:209-211.

Hurst JA, Baraitser M, Auger E, Graham F, Norell S (1990) An extended family with a dominantly inherited speech disorder. Dev Med Child Neurol 32:352-355.

Jarvis ED, Nottebohm F (1997) Motor-driven gene expression. Proc Natl Acad Sci USA 94:4097-4102.

Jarvis ED, Scharff C, Grossman MR, Ramos JA, Nottebohm F (1998) For whom the bird sings: context-dependent gene expression. Neuron 21:775-788.

Kao MH, Doupe AJ, Brainard MS (2005) Contributions of an avian basal ganglia-forebrain circuit to real-time modulation of song. Nature 433:638-643.

Lai CS, Fisher SE, Hurst JA, Vargha-Khadem F, Monaco AP (2001) A forkhead-domain gene is mutated in a severe speech and language disorder. Nature 413:519-523.

Lai CS, Gerrelli D, Monaco AP, Fisher SE, Copp AJ (2003) FOXP2 expression during brain development coincides with adult sites of pathology in a severe speech and language disorder. Brain 126:2455-2462.

Li S, Weidenfeld J, Morrisey EE (2004) Transcriptional and DNA binding activity of the Foxp1/2/4 family is modulated by heterotypic and homotypic protein interactions. Mol Cell Biol 24:809-822.

Lieberman P (2002) On the nature and evolution of the neural bases of human language. Am J Phys Anthropol [Suppl] 35:36-62.

Liegeois F, Baldeweg T, Connelly A, Gadian DG, Mishkin M, Vargha-Khadem F (2003) Language fMRI abnormalities associated with FOXP2 gene mutation. Nat Neurosci 6:1230-1237.
Livingston FS, White SA, Mooney R (2000) Slow NMDA-EPSCs at synapses critical for song development are not required for song learning in zebra finches. Nat Neurosci 3:482-488.

Lu MM, Li S, Yang H, Morrisey EE (2002) Foxp4: a novel member of the Foxp subfamily of winged-helix genes co-expressed with Foxp1 and Foxp2 in pulmonary and gut tissues. Mech Dev 119 [Suppl 1]:S197-S202.

MacDermot KD, Bonora E, Sykes N, Coupe AM, Lai CS, Vernes SC, VarghaKhadem F, McKenzie F, Smith RL, Monaco AP, Fisher SE (2005) Identification of FOXP2 truncation as a novel cause of developmental speech and language deficits. Am J Hum Genet 76:1074-1080.

Mello CV, Vicario DS, Clayton DF (1992) Song presentation induces gene expression in the songbird forebrain. Proc Natl Acad Sci USA 89:6818-6822.

Nottebohm F (1981) A brain for all seasons: cyclical anatomical changes in song control nuclei of the canary brain. Science 214:1368-1370.

Olveczky BP, Andalman AS, Fee MS (2005) Vocal experimentation in the juvenile songbird requires a basal ganglia circuit. PLoS Biol 3:e153.

Scharff C, Haesler S (2005) An evolutionary perspective on FoxP2: strictly for the birds? Curr Opin Neurobiol 15:694-703.

Shu W, Yang H, Zhang L, Lu MM, Morrisey EE (2001) Characterization of a new subfamily of winged-helix/forkhead (Fox) genes that are expressed in the lung and act as transcriptional repressors. J Biol Chem 276:27488-27497.

Shu W, Cho JY, Jiang Y, Zhang M, Weisz D, Elder GA, Schmeidler J, De Gasperi R, Sosa MA, Rabidou D, Santucci AC, Perl D, Morrisey E, Buxbaum JD (2005) Altered ultrasonic vocalization in mice with a disruption in the Foxp2 gene. Proc Natl Acad Sci USA 102:9643-9648.

Sohrabji F, Nordeen EJ, Nordeen KW (1990) Selective impairment of song learning following lesions of a forebrain nucleus in the juvenile zebra finch. Behav Neural Biol 53:51-63.

Tchernichovski O, Nottebohm F, Ho CE, Pesaran B, Mitra PP (2000) A procedure for an automated measurement of song similarity. Anim Behav 59:1167-1176.

Teramitsu I, Kudo LC, London SE, Geschwind DH, White SA (2004) Parallel FoxP1 and FoxP2 expression in songbird and human brain predicts functional interaction. J Neurosci 24:3152-3163.

Thompson CK, Brenowitz EA (2005) Seasonal change in neuron size and spacing but not neuronal recruitment in a basal ganglia nucleus in the avian song control system. J Comp Neurol 481:276-283.

Vargha-Khadem F, Gadian DG, Copp A, Mishkin M (2005) FOXP2 and the neuroanatomy of speech and language. Nat Rev Neurosci 6:131-138.

Watkins KE, Dronkers NF, Vargha-Khadem F (2002) Behavioural analysis of an inherited speech and language disorder: comparison with acquired aphasia. Brain 125:452-464.

Zann RA (1996) The zebra finch: a synthesis of field and laboratory studies, pp 157-246. New York: Oxford UP.

Zeesman S, Nowaczyk MJ, Teshima I, Roberts W, Cardy JO, Brian J, Senman L, Feuk L, Osborne LR, Scherer SW (2006) Speech and language impairment and oromotor dyspraxia due to deletion of $7 \mathrm{q} 31$ that involves FOXP2. Am J Med Genet A 140:509-514. 